\title{
Fast Deterministic Consensus in a Noisy Environment
}

\author{
James Aspnes*
}

June 3, 2018

\begin{abstract}
It is well known that the consensus problem cannot be solved deterministically in an asynchronous environment, but that randomized solutions are possible. We propose a new model, called noisy scheduling, in which an adversarial schedule is perturbed randomly, and show that in this model randomness in the environment can substitute for randomness in the algorithm. In particular, we show that a simplified, deterministic version of Chandra's wait-free shared-memory consensus algorithm (PODC, 1996, pp. 166-175) solves consensus in time at most logarithmic in the number of active processes. The proof of termination is based on showing that a race between independent delayed renewal processes produces a winner quickly. In addition, we show that the protocol finishes in constant time using quantum and priority-based scheduling on a uniprocessor, suggesting that it is robust against the choice of model over a wide range.
\end{abstract}

\section{Introduction}

Perhaps the single most dramatic result in the theory of distributed computing is Fischer, Lynch, and Paterson's proof of the impossibility of deterministic consensus in an asynchronous environment with failures [22]. This result and its extensions [20,27] show that the consensus problem, in which a group of processes must collectively agree on a bit, cannot be solved deterministically in an asynchronous message-passing or shared-memory model

\footnotetext{
* Yale University, Department of Computer Science, 51 Prospect Street/P.O. Box 208285, New Haven CT 06520-8285. Email: aspnes@cs.yale.edu. This work was supported in part by NSF grants CCR-9820888 and CCR-0098078.
} 
if an unrestricted adversary controls scheduling. Solutions to the sharedmemory version of this fundamental problem have thus taken the approach of restricting the adversary, either by allowing randomization that limits the adversary's knowledge [1, 6, 8, 10, 12, 13, 15 17, 30] or by imposing timing constraints that limit the adversary's control [3, 20, 21]. As a corollary to granting less power to the adversary, these solutions often involve granting more power to the algorithm, in the form of the ability to obtain random bits or explicitly delay steps. By using these additional powers an algorithm can escape the FLP bound and reach agreement.

These additional powers come at a cost. Randomization alone is not powerful enough to allow sublinear consensus protocols [7], so efficient randomized solutions have required additional constraints on the ability of the adversary to observe the arguments to operations and the contents of unread memory locations 12, 13, 16. These algorithms carefully manage common pools of unread random bits for future use, a clever but odd-looking practice that is justified primarily by the specific details of the model. The delay-based algorithm of [3] is less convoluted, but still depends on using explicit delays that at the minimum require that a process has the power to invoke them and at worst may add unnecessary delay when few processes participate.

As an alternative to designing an algorithm specifically to exploit the weaknesses of a particular adversary model, we consider the approach of using a simple algorithm that guarantees agreement but relies on good luck to terminate. Our LEAN-CONSENSUS algorithm, described in Section 1 , is obtained by removing all of the randomized parts of a similar algorithm due to Chandra [16]. The essential idea (which is the core of many consensus protocols in the literature) is to stage a race between those processes that prefer 0 and those that prefer 1, with the rule that if a slow process sees that faster processes are all in agreement it adopts their common preference. The race is implemented using two arrays of atomic read/write bits. The algorithm terminates when the fastest processes are all in agreement and can decide on their preferred value safely, knowing that other processes will adopt the same preference before they catch up. As shown in Section 5 , this mechanism is enough to ensure that if any one process decides then all other processes soon decide on the same value, no matter how the adversary arranges the schedule.

In effect, the race framework allows the processes to detect agreement once it occurs. But unlike other consensus algorithms, LEAN-CONSENSUS makes no attempt to cajole the processes into reaching agreement- it relies entirely on the hope that some process eventually pulls ahead of the others. 
In order to dash this hope, the adversary must exercise enough control to ensure that the fastest processes run in lockstep. We believe that in many natural system models it will be difficult for the adversary to exercise this much control.

One such model is what we call the noisy scheduling model, described in Section 3.1. In this model, the adversary proposes a schedule that specifies the order in which read and write operations occur, but this schedule is perturbed by random noise drawn from some arbitrary non-constant distribution. This noise corresponds to random factors in a system that might not be strongly correlated with the algorithm's behavior, such as network delays, clock skew, or bus or memory contention.

We show in Section 6 that, in the noisy scheduling model, LEAN-CONSENSUS terminates with expected $\Theta(\log n)$ work per process, where $n$ is the number of active processes. This result is distribution-independent, in the sense that the algorithm's asymptotic performance does not depend on the noise distribution in the model (though the constant factor does), and it holds even if processes are subject to random halting failures. Because the algorithm's performance depends only on the number of processes actually executing the protocol and not on the total number of processes in the system, it is adaptive in the sense of [11], which implies it is fast in the sense of [2, 26]. Thus it is well-suited to situations where only one or a few processes attempt to run the algorithm at the same time.

Our noisy scheduling model is similar to the model used by Gafni and Mitzenmacher [23] in their analysis of mutual exclusion protocols with random timing, but is extended to include constant delays inserted by the adversary in addition to random delays. Another source of inspiration is Koutsoupias and Papadimitriou's diffuse adversary [25], which chooses a distribution over executions in which no branch at any decision point can occur with probability more than some fixed $\epsilon$. Our model is not the first in which an adversary chooses parameters for a stochastic process that then controls scheduling; a sophisticated model of this type, based on asynchronous PRAMs, has been proposed by Cole and Zajicek [19].

To give support to our intuition that many possible restrictions on the adversary make LEAN-CONSENSUS work, we also consider what happens with a hybrid quantum and priority-based scheduler on a uniprocessor, following the approach of [5]. (The details of this model, which subsumes both quantum scheduling and priority-based scheduling, are sketched in Section 3.2.) We show in Section 07 that LEAN-CONSENSUs terminates in $O(1)$ steps in the hybrid-scheduling model, as long as the quantum is at least 8 . The restriction to a uniprocessor is necessary because [5] shows that no deter- 
ministic algorithm can solve consensus with multiple processors, even with hybrid scheduling, without using stronger primitives than atomic read/write registers.

Our basic consensus algorithm requires infinitely long arrays. Obviously this is undesirable in a real system. In order to bound the required space, we adopt a technique from [16] and cut off the algorithm after consuming $O\left(\log ^{2} n\right)$ bits of space, using the preference each undecided process has at that point as input to a more expensive, bounded-memory consensus algorithm satisfying the validity property Since the more expensive algorithm is only run with low probability, its higher costs do not increase the expected time for the algorithm as a whole by more than a small constant factor. Details are given in Section 8 .

Section 9 describes some simulation results that show that the constant factors in the noisy scheduling analysis are in fact quite small for plausible noise distributions, suggesting that the good theoretical performance of LEAN-CONSENSUS might actually translate into fast execution in a real system.

In Section 10, we suggest a number of directions in which the current work could be extended, including extensions to the noisy scheduling model. One interesting possibility is the inclusion of adaptive crash failures. We argue briefly that because LEAN-CONSENSUS recovers quickly from such failures, it terminates in at most $O(f \log n)$ work per process even if up to $f$ processes fail. However, there remains an interesting open question whether noisy scheduling is enough to get $O(\log n)$ performance even with $\Theta(n)$ crash failures.

\section{The Consensus Problem}

In the binary consensus problem, a group of $n$ processes, possibly subject to halting failures, must agree on a bit.2 A consensus protocol is a distributed algorithm in which each non-faulty process starts with an input bit and eventually terminates by deciding on an output bit. It must satisfy the following three conditions with probability 1 :

\footnotetext{
${ }^{1}$ An early example of this approach is found in the bounded-rounds randomized Byzantine agreement protocol of Goldreich and Petrank 24], which switches from a randomized to a deterministic protocol if the randomized protocol does not terminate quickly enough.

${ }^{2}$ Some authors consider the stronger problem of $i d$ consensus, in which the decision value is the id of some active process. In many cases, id consensus can be solved in a natural way using a $(\lg n)$-depth tree of binary consensus protocols; examples of this approach can be found in [12, 16].
} 
- Agreement. All non-faulty processes decide on the same bit.

- Termination. All non-faulty processes finish the protocol in a finite number of steps.

- Validity. If all processes start with the same input bit, all non-faulty processes decide on that bit. [?

\section{Model}

We assume a shared-memory system consisting of an unbounded number of processes that communicate only through shared atomic read/write registers. We use the usual interleaving model, in which operations are assumed to occur in a sequence $\pi_{1}, \pi_{2}, \ldots$, and in which each read operation returns the value of the last previous write to the same location. The order in which operations occur is determined by a stochastic process that is partially under the control of an adversary (Section 3.1), or directly by the adversary subject to certain regularity constraints (Section 3.2).

\subsection{Noisy Scheduling}

In the noisy scheduling model, we assume that the adversary specifies when operations occur (subject to an upper bound on the time between successive operations by the same process), but that this specification is perturbed by random noise.

Formally, the adversary chooses:

1. An arbitrary starting time $\Delta_{i 0}$ for each process $p_{i}$,

2. A non-negative delay $\Delta_{i j}$ between process $p_{i}$ 's $(j-1)$-th and $j$-th operations, bounded by some fixed constant $M$, and

3. A fixed common distribution $F_{\pi}$ of the random delay added to each type of operation $\pi$ (e.g., read or write). If process $p_{i}$ 's $j$-th operation is of type $\pi$, it suffers an additional delay $X_{i j}$ whose distribution is $F_{\pi}$. There is no restriction on the choice of the $F_{\pi}$, except that they must

\footnotetext{
${ }^{3}$ Some definitions of consensus replace the validity condition with a weaker nontriviality condition that says that there must exist executions in which different decision values occur.
} 
not be concentrated on a point and must produce only non-negative values $X_{i j}$. 田

The time of process $p_{i}$ 's $j$-th operation is given by

$$
S_{i j}=\Delta_{i 0}+\sum_{k=1}^{j}\left(\Delta_{i k}+X_{i k}\right) .
$$

Since we are using interleaving semantics, the effect of executing two operations at exactly the same time is not well-defined. To avoid ill-defined executions, we impose the additional technical constraint on the adversary's choices that the probability that any two operations occur simultaneously must be zero. This is automatic if, for example, the noise distributions $F_{\pi}$ are continuous. Alternatively, it can be arranged by dithering the starting times of each process by some small epsilon. This technical constraint does not qualitatively change our results.

Below we discuss the unfairness of noisy scheduling and extensions to allow random failures.

\subsubsection{Unfairness}

The upper bound on the $\Delta_{i j}$ and the common distribution on the $X_{i j}$ might suggest that the noisy scheduling model produces fair schedules. This is not entirely true for sufficiently pathological distributions.

Theorem 1 There exists a choice of $F_{\pi}$ and $\Delta_{i j}$ such that for any distinct processes $p_{i}$ and $p_{i^{\prime}}$, and any operation $j$, the expected number of operations $p_{i^{\prime}}$ completes between $p_{i}$ 's $j$-th and $(j+1)$-th operations is infinite.

Proof: Set each $F_{\pi}$ so that $X_{i j}$ takes on the value $2^{k^{2}}$ with probability $2^{-k}$ for $k=1,2, \ldots$. For simplicity, let us suppose that $\Delta_{i j}=0$ for $j>0$. We will also assume that $A$ and $B$ execute no operations before time 0 .

Let $X$ be the number of operations completed by $p_{i^{\prime}}$ between $S_{i j}$ and $S_{i, j+1}$. We will show that the expectation of $X$ is infinite conditioned on the value of $t=\left\lceil S_{i j}\right\rceil$ (the ceiling is so that we have countably many cases).

The idea is this: for each $k$ we have probability $2^{-k}$ that $S_{i, j+1} \geq$ $X_{i, j+1}=2^{k^{2}}$. Condition on this event occurring for some particular $k$ and consider how many operations $p_{i^{\prime}}$ must execute to reach time $2^{k^{2}}$. Either (a) one of these operations takes time $2^{k^{2}}$ or more (with probability $2^{-k+1}$

\footnotetext{
${ }^{4}$ In fact, the $F_{\pi}$ distributions can be quite bizarre; it is not required, for example, that the $X_{i j}$ have finite expectation.
} 
per operation); or (b) a total of at least $2^{2 k-1}$ faster operations, each of which takes at most $2^{(k-1)^{2}}$ time, must occur. If we wait only for event (a), we expect to see $2^{k-1}$ operations; to get the actual expected number, we must subtract off the expected number of operations until (a) occurs after (b) occurs $\left(2^{k-1}\right.$ again) multiplied by the probability that (b) occurs. This latter probability is at most $\left(1-\frac{1}{2^{k-1}}\right)^{2 k-1}$, which goes to $e^{-2}$ in the limit as $k$ grows; it follows that $p_{i^{\prime}}$ executes $\Omega\left(2^{k}\right)$ operations on average before time $2^{k^{2}}$. Of these, at most $t / 2$ can occur before time $S_{i j}$, so if $k \gg \lg t$, we have $\Omega\left(2^{k}\right)$ operations on average between $t$ and $2^{k^{2}}$, and thus also between $S_{i j}$ and $S_{i, j+1}$, since $S_{i j} \leq t<2^{k^{2}} \leq S_{i, j+1}$.

To get the full result, we must remove two layers of conditioning. First compute the expectation conditioned only on $t$ by summing $2^{-k} \Omega\left(2^{k}\right)$ for each of the infinitely many sufficiently large $k$. It is not difficult to see that this sum diverges and the expectation is infinite. Summing over all values of $t$ doesn't make it any less infinite, and we are done.

\subsubsection{Failures}

We can extend the noisy scheduling model to allow halting failures. For each $i$ and each $j>0$ let $H_{i j}=\infty$ if process $p_{i}$ halts before its $j$-th operation and 0 otherwise. Define

$$
S_{i j}^{\prime}=\Delta_{i 0}+\sum_{k=1}^{j}\left(\Delta_{i k}+X_{i k}+H_{i k}\right),
$$

with the usual convention for the extended real line that $x+\infty=\infty+x=\infty$ for any finite $x$. If $S_{i j}^{\prime}=\infty, p_{i}$ 's $j$-th operation does not occur.

We do not include failures in the noise distributions $F_{\pi}$ because these distributions do not depend on $n$, and a constant probability of failure would mean that all processes die after $O(\log n)$ steps. Instead, we assume that failures occur independently with probability $h(n)$ per operation, where $h$ is some function chosen by the adversary. The effect of stronger failure models is discussed in Section 10.

\subsection{Quantum and Priority-Based Scheduling}

Our intuition is that LEAN-CONSENSUS should perform well in any setting that prevents lockstep executions. One such setting is the hybrid-scheduled uniprocessor model of [5], which combines the priority-based scheduling model of 29] with the quantum-based scheduling model of 四. In this model, 
processes are assumed to be time-sharing a uniprocessor under the control of a pre-emptive scheduler. Each process has a priority, and a process may be pre-empted at any time by a process of higher priority. A process may only be pre-empted by a process of the same priority if it has exhausted its quantum, a minimum number of operations it must complete between the time it wakes up and the time at which it becomes vulnerable to preemption. There is no requirement that a process start the protocol at the beginning of a quantum; it may have used up some or all of its quantum performing other work before starting the protocol. We do not consider failures in the hybrid-scheduling model; instead, a process may be arbitrarily delayed subject to the constraints on the scheduler.

\section{The LEAn-COnsensus Algorithm}

In this section, we describe the LEAN-CONSENSUS algorithm. The algorithm is very simple, because we are relying on randomness in the environment to guarantee termination and thus the algorithm itself must only guarantee correctness and provide the opportunity for the underlying system to quickly jostle it into a decision state. Structurally, it is essentially identical to the multi-writer register consensus protocol of Chandra [16] with the shared coins removed, leaving only the implementation from multi-writer bits of the "racing counters" technique that has been used in many shared-memory consensus protocols. It also bears some similarities to the Time-Adaptive Consensus algorithm of Alur et al. [3] with the delays removed.

At each step of the algorithm, each process prefers either 0 or 1 as its decision value. The conflict between the 0 -preferring processes and the 1preferring processes is settled by a race implemented using two arrays $a_{0}$ and $a_{1}$ of atomic read/write bits, each initialized to zero. Each process carries out a sequence of rounds, each consisting of a fixed sequence of operations. During round $r$, a process that prefers $b$ marks location $a_{b}[r]$ with a one and looks to see if either (a) it has fallen behind its rivals who prefer $(1-b)$, in which case it abandons its former preference and joins the winning team, or (b) it and its fellows have sped far enough ahead of any rival processes that they can safely decide $b$ knowing that those rivals will give up and join the $b$ team before they catch up. The algorithm finishes fastest when the pack of processes disperses quickly, so that a clear winner emerges as early as possible.

Let us look more closely at the details of the algorithm. A process with input $b$ sets its preference $p$ to $b$ and its round number $r$ to 1 . (We say that 
a process is at round $r$ if its round number is set to $r$; processes thus start at round 1.) It then repeatedly executes the following sequence of steps. To simplify the description of the algorithm, we assume that while $a_{0}$ and $a_{1}$ are initialized to zeroes, they are prefixed with (effectively read-only) locations $a_{0}[0]$ and $a_{1}[0]$, both set to 1 .

1. Read $a_{0}[r]$ and $a_{1}[r]$. If for some $b, a_{b}[r]$ is 1 and $a_{1-b}[r]$ is 0 , set $p$ to $b$.

2. Write 1 to $a_{p}[r]$.

3. Read $a_{1-p}[r-1]$. If this value is 0 , decide $p$ and exit.

4. Otherwise, set $r$ to $r+1$ and repeat.

Note that in each round the process carries out exactly four operations in the same sequence: two reads, a write, and another read. It is tempting to optimize the algorithm by eliminating the write when it is already evident from the previous step that $a_{p}[r]$ is set or eliminating the last read when it can be deduced from the value of $a_{1-p}[r]$ that $a_{1-p}[r-1]$ is set. However, this optimization reduces the work done by slow processes (whom we'd like to have fall still further behind) while maintaining the same per-round cost for fast processes (whom we'd like to have pull ahead). So we must paradoxically carry out operations that might appear to be superfluous in order to minimize the actual total cost.

\section{$5 \quad$ Agreement and Validity}

If we ignore the termination requirement, the correctness of the algorithm does not depend on the behavior of the scheduler. The following two lemmas show that the validity and agreement properties hold whenever the algorithm terminates. The proofs are very similar in spirit to those of Lemmas 1-4 in [16].

Lemma 2 No process sets $a_{b}[r]$ unless (a) $r=1$ and $b$ is an input value, or (b) $r>1$ and $a_{b}[r-1]$ has already been set.

Proof: Consider the first process $P$ that sets $a_{b}[r]$. Then $P$ does not read 1 from $a_{b}[r]$ at round $r$ and does not change its preference during round $r$. If $r=1, P$ 's preference equals its input, establishing case (a); if $r>1, P$ must have set $a_{b}[r-1]$ at round $r-1$, establishing case (b). 
Lemma 3 If every process starts with the same input bit b, every process decides $b$ after executing 8 operations.

Proof: From Lemma 2, if no process has input $1-b$, no process ever sets $a_{1-b}[1]$. It follows that every process sees a zero in $a_{1-b}$ [1] at round 2 and decides $b$.

Lemma 4 If some process decides $b$ at round $r$, then (a) no process ever writes $a_{1-b}[r]$, and (b) every process decides $b$ at or before round $r+1$.

Proof: Let $P$ decide $b$ at round $r$. We will show that this implies that no process ever sets $a_{1-b}[r]$.

Suppose some process sets $a_{1-b}[r]$; let $Q$ be the first such process. Because $Q$ is the first process to set $a_{1-b}[r]$, it must read a 0 from $a_{1-b}[r]$ at the start of round $r$. Thus $Q$ can only set $a_{1-b}[r]$ if it already prefers $1-b$ at the start of round $r$, implying that it set $a_{1-b}[r-1]$ during round $r-1$; and if it reads a 0 from $a_{b}[r]$ at the start of round $r$, preventing it from changing its preference after seeing a 0 in $a_{1-b}[r]$. But $Q$ 's read of $a_{b}[r]$ occurs after $Q$ 's write to $a_{1-b}[r-1]$, which occurs after $P$ 's read of $a_{1-b}[r-1]$ at round $r$ (because $P$ reads 0 ), which in turn occurs after $P$ 's write to $a_{b}[r]$. Thus $Q$ reads 1 from $a_{b}[r]$, and changes its preference to $b$ at round $r$. This contradicts our assumption that $Q$ is the first to set $a_{1-b}[r]$. It follows that if any process decides $b$ in round $r$, no process sets $a_{1-b}[r]$.

Since no process sets $a_{1-b}[r]$, any process that reaches round $r+1$ must set $a_{b}[r+1]$ (by Lemma 2), and will decide $b$ after reading 0 from $a_{1-b}[r]$. Thus no process runs past round $r+1$ without deciding $b$.

To show agreement in earlier rounds, let $P^{\prime}$ decide $b^{\prime}$ at round $r^{\prime} \leq r$. By the preceding argument, if $P^{\prime}$ decides $b^{\prime}$ at round $r^{\prime}$, then no process sets $a_{1-b^{\prime}}\left[r^{\prime}\right]$ and thus (by Lemma 2 again) no process sets $a_{1-b^{\prime}}[r]$. But since $P$ sets $a_{b}[r]$, we must have $b^{\prime}=b$.

\section{Termination with Noisy Scheduling}

In this section, we show that LEAN-CONSENSUS terminates in $\Theta(\log n)$ rounds with noisy scheduling and random failures. (This analysis includes the core model without random failures as well, since the adversary can always choose $h(n)=0$.) We show that either all processes die (in which case we treat the algorithm as terminating in the last round in which some process takes a step), or some group of processes with a common preference eventually gets 
two rounds ahead of the other processes. To avoid analyzing the details of how processes shift preferences, we will show the even stronger result that unless all processes die, a single process eventually gets two rounds ahead of the other processes.

To simplify the argument, we abstract away from the individual sequence of operations in each round and look only at the times at which rounds are completed. We can thus assume that the adversary provides a single noise distribution $F$ (corresponding to the distribution of the sum of the delays on three reads and one write) and that the values $\Delta_{i j}, X_{i j}$, and $H_{i j}$ provide the delay not on the $j$-th operation but on the $j$-th round. Since this abstraction merely involves summing together the underlying variables on operations, it does not reduce the adversary's control over the protocol. We will scale $M$ appropriately so that it is still the case that $0 \leq \Delta_{i j} \leq M$ when $j>0$.

Using this approach, the increment $\Delta_{i j}+X_{i j}+H_{i j}$ is the time taken for process $i$ to move from the end of round $j-1$ to the end round $j$. The constant $\Delta_{i 0}$ represents the process's starting time, and $S_{i r}^{\prime}=\Delta_{i 0}+$ $\sum_{j=1}^{r}\left(\Delta_{i j}+X_{i j}+H_{i j}\right)$ gives the time at which the process finishes round $r$. A process $i$ wins the race with a lead of $c$ rounds at round $r+c$ if it finishes round $r+c$ before any other process finishes round $r$, i.e., if $S_{i, r+c}^{\prime} \leq S_{i^{\prime}, r}^{\prime}$ for all $i^{\prime} \neq i$.

We would like to show a bound on how the expected round at which some process wins by $c$ scales as a function of the number of processes $n$, keeping $c$, $M$, and $F$ fixed. This bound is given in Corollary 11 below. We will assume that $h(n)=o(1)$, as otherwise all processes die after $O(\log n)$ rounds on average. The proof proceeds in two steps: first we show that for any $r$ which some process finishes with at least constant probability, there exists a critical time $t$ that gives at least a constant probability that $S_{i r}^{\prime} \leq t$ for exactly one $i$. We then show that if $r$ is large enough, $\operatorname{Pr}\left[S_{i, r+c}^{\prime} \leq t \mid S_{i r}^{\prime} \leq t\right]$ is also at least a constant. It then follows that the probability that $S_{i, r+c}^{\prime} \leq t$ while $S_{i^{\prime} r}^{\prime}>t$ for any $i^{\prime} \neq i$ is at least the product of these two constants and the constant probability that $p_{i}$ is not killed between rounds $r$ and $r+c$. Thus after a constant number of phases each consisting of $r+c$ rounds we expect some process to win.

\subsection{Existence of a winner}

In this section, we build up the tools needed to show that for each round there exists a fixed time at which there is likely to be a unique winner. 
Lemma 5 Let $A_{1}, \ldots, A_{n}$ be independent events. If the probability that no $A_{i}$ occurs is $x$, where $x$ is not zero, then the probability that exactly one $A_{i}$ occurs is at least $-x \ln x$.

Proof: Let $q_{i}$ be the probability that $A_{i}$ does not occur. The probability $x$ that no $A_{i}$ occurs is the product of the $q_{i}$. Since $x$ is nonzero, each $q_{i}$ must also be nonzero. The probability that exactly one $A_{i}$ occurs is given by

$$
\begin{aligned}
\left(\prod_{i=1}^{n} q_{i}\right) \sum_{i=1}^{n} \frac{1-q_{i}}{q_{i}} & =x \sum_{i=1}^{n}\left(\frac{1}{q_{i}}-1\right) \\
& =x\left(-n+\sum_{i=1}^{n} \frac{1}{q_{i}}\right) .
\end{aligned}
$$

Let $G$ be the geometric mean of the $q_{i}$ and let $H$ be their harmonic mean. By the theorem of the means, $G>H$. Observe that $G=x^{1 / n}$ and

$\sum_{i=1}^{n} \frac{1}{q_{i}}=n / H>n / G=n x^{-1 / n}=n \exp \left(-\frac{\ln x}{n}\right) \geq n\left(1-\frac{\ln x}{n}\right)=n-\ln x$.

Plugging this inequality into (11) gives the result.

Suppose $X_{1}, \ldots, X_{n}$ are random times. The following lemma shows that under certain conditions there exists a constant time $t_{0}$, such that, with constant probability, at most one of the $X_{i}$ is less than $t_{0}$ :

Lemma 6 Let $X_{1}, \ldots, X_{n}$ be independent random variables such that for all finite values $t$ and all distinct $i, j$, the probability that $X_{i}=X_{j}=t$ is zero. Then either $\operatorname{Pr}\left[\forall i X_{i}=\infty\right]$ is greater than $e^{-1}$ or there exists $t_{0}$ such that the probability that exactly one of the $X_{i}$ is less than or equal to $t_{0}$ is at least $1 / 5$.

Proof: For each $t$, let $q_{i}(t)$ be the probability that $X_{i}$ is not less than or equal to $t$. Let $q(t)=\prod_{i=1}^{n} q_{i}(t)$ be the probability that none of the $X_{i}$ are less than or equal to $t$. Note that each $q_{i}(t)$ is a decreasing rightcontinuous left-limited function with $\lim _{t \rightarrow-\infty} q_{i}(t)=1$ and $\lim _{t \rightarrow \infty} q_{i}(t)=$ $\operatorname{Pr}\left[X_{i}=\infty\right]$. Similarly, $q(t)=\prod_{i} q_{i}(t)$ is right-continuous, left-limited, and has $\lim _{t \rightarrow-\infty} q(t)=1$ and $\lim _{t \rightarrow \infty} q(t)=\operatorname{Pr}\left[\forall i X_{i}=\infty\right]$.

Suppose that this latter quantity is less than or equal to $e^{-1}$. (If not, the first case of the lemma holds.) Then for some finite $t, q(t) \leq e^{-1}$. Let $t_{0}$ be the least such $t$. 
Now suppose $q\left(t_{0}\right) \geq e^{-2}$. Then, by Lemma 5 , the probability that exactly one $X_{i}$ is less than or equal to $t_{0}$ is at least $2 e^{-2} \approx 0.27 \ldots$.

Otherwise, we have $q\left(t_{0}\right)<e^{-2}$ but $q\left(t_{0}-\right)=\lim _{t \rightarrow t_{0}-} q(t)>e^{-1}$. (We are using the usual convention that $f(x-)$ denotes the left limit of $f$ at $x$.) This discontinuity must correspond to a discontinuity in $q_{i}$ for some $i$. At most one $q_{i}$ has a discontinuity at $t_{0}$, by the assumption that the probability that distinct $X_{i}, X_{j}$ both equal $t_{0}$ is zero. Hence, for all $j \neq i$ we have $q_{j}\left(t_{0}-\right)=q_{j}\left(t_{0}\right)$ and thus $q_{i}\left(t_{0}-\right) / q_{i}\left(t_{0}\right)=q\left(t_{0}-\right) / q\left(t_{0}\right) \leq e^{-1}$.

Since $q_{i}\left(t_{0}-\right) \leq 1$, it follows immediately that $q_{i}\left(t_{0}\right) \leq e^{-1}$ and thus the probability that $X_{i}$ is less than or equal to $t_{0}$ is at least $1-e^{-1}$. Now the probability that no other $X_{j}$ is less than or equal to $t_{0}$ is at least $q\left(t_{0}\right) / q_{i}\left(t_{0}\right) \geq q\left(t_{0}-\right)>e^{-1}$. Since the variables are independent, the probability that only $X_{i}$ is less than or equal to $t_{0}$ is thus at least $\left(1-e^{-1}\right) e^{-1} \approx 0.23 \ldots$

\subsection{Size of the lead}

In this section, we show that if enough rounds have passed, a process that is likely to be ahead of the others is in fact likely to be several rounds ahead. The proof is somewhat complicated by the lack of restrictions on the noise distribution, but the following lemma shows how the Strong Law of Large Numbers can be used to smooth the noise terms out a bit.

Lemma 7 Let $X_{1}, X_{2}, \ldots$ be finite non-negative independent identically distributed random variables whose common distribution is not concentrated on a point. Define $S_{n}=\sum_{i=1}^{n} X_{i}$. For any $c$, there exist $n, t$ such that $\operatorname{Pr}\left[S_{n}<t\right]<\frac{1}{2}$ but $\operatorname{Pr}\left[S_{n}<t-c\right]>0$.

Proof: Let us first consider the case where $X_{i}$ has a finite expectation $m$. Then the Strong Law of Large Numbers says that $S_{n} / n$ converges to $m$ in the limit with probability 1 . So for any $\epsilon>0$, the probability that $S_{n}$ is less than $m-\epsilon$ goes to zero and thus drops below $1 / 2$ for all $n$ greater than some $n_{0}$.

Let $t_{n}=n(m-\epsilon)$. As long as $n>n_{0}$, we have $\operatorname{Pr}\left[S_{n}<t\right]<\frac{1}{2}$. Now suppose that $\operatorname{Pr}\left[S_{n}<t_{n}-c\right]=0$ whenever $n>n_{0}$. Since the $X_{i}$ are independent, this event can only occur if for each $X_{i}, X_{i}<\frac{t_{n}-c}{n}=m-\epsilon-\frac{c}{n}$ with probability 0 . Taking the union of countably many such bad events for each rational $\epsilon$ and each $n>n_{0}$ shows that the event $X_{i}<m$, also has probability 0 . It follows that $X_{i} \geq E\left[X_{i}\right]$ almost surely and thus the distribution of $X_{i}$ is concentrated on $E\left[X_{i}\right]$, a contradiction. 
If $X_{i}$ does not have a finite expectation, then $S_{n} / n$ grows without bound with probability 1 (see the corollary to Theorem 22.1 in 14]). So for any $x$, there exists $n_{0}$, such that $\operatorname{Pr}\left[S_{n} / n<x\right]<\frac{1}{2}$ for $n>n_{0}$. We repeat the above analysis for $t=n x$; if $\operatorname{Pr}\left[S_{n}<t-c\right]=0$ for all such $t$, we get $X_{i} \geq x-\frac{c}{n}$ almost surely, implying $X_{i}$ exceeds any finite bound $x$. Again, a contradiction.

Once the noise terms have been smoothed, it is not hard to show that they eventually accumulate enough to push a winner ahead:

Lemma 8 Fix $c>0$. Let $X_{1}, X_{2}, \ldots$ be finite independent identically distributed random variables such that there exists a threshold $t_{0}$ for which $\operatorname{Pr}\left[X<t_{0}\right]<\frac{1}{2}$ but $\operatorname{Pr}\left[X<t_{0}-c\right]=\delta_{0}>0$. Define $S_{n}=\sum_{i=1}^{n} X_{i}$.

Then for any $\epsilon>0$, there exists an $n=O(\log (1 / \epsilon))$, such that for any $t, \operatorname{Pr}\left[S_{n}<t\right]>\epsilon$ implies $\operatorname{Pr}\left[S_{n}<t-c \mid S_{n}<t\right]>\frac{1}{7} \delta_{0}$.

Proof: Set $n=8(\ln (1 / \epsilon)+1)$. Each $X_{i}$ has probability at most $1 / 2$ of being less than $t_{0}$, so a simple application of Chernoff bounds shows that the probability that $3 / 4$ or more of the $X_{i}$ are less than $t_{0}$ is at most $e^{-n / 8}=\epsilon / e$.

We will use this fact to argue that even when conditioning on $S_{n}<t$, there is nearly one chance in four that $X_{n}$ in particular is greater than $t_{0}$. In this case, $S_{n-1}$ is less than $t-t_{0}$ and we can use independence to replace $X_{n}$ with a new value less than $t_{0}-c$, giving a sum $S_{n}$ less than $t-c$, all without reducing the probability by much.

Formally, we have the following sequence of inequalities, each of which is implied by the previous one. Let $\operatorname{Pr}\left[S_{n}<t\right]=p$ and suppose $p>\epsilon$. Then we have:

$$
\begin{aligned}
\operatorname{Pr}\left[S_{n}<t\right] & =p \\
\operatorname{Pr}\left[S_{n}<t \wedge \text { at least } \frac{1}{4} \text { of } X_{i} \text { are greater than } t_{0}\right] & >p-\epsilon / e \\
\operatorname{Pr}\left[S_{n}<t \wedge X_{n}>t_{0}\right] & >\frac{1}{4}(p-\epsilon / e) \\
\operatorname{Pr}\left[S_{n-1}<t-t_{0}\right] & >\frac{1}{4}(p-\epsilon / e) \\
\operatorname{Pr}\left[S_{n-1}<t-t_{0} \wedge X_{n}<t_{0}-c\right] & >\frac{1}{4}(p-\epsilon / e) \delta_{0} \\
\operatorname{Pr}\left[S_{n}<t-c\right] & >\frac{1}{4}(p-\epsilon / e) \delta_{0} \\
\operatorname{Pr}\left[S_{n}<t-c \mid S_{n}<t\right] & >\frac{1}{4}(p-\epsilon / e) \delta_{0} / p
\end{aligned}
$$


Since $p>\epsilon$, this last quantity is at least $\frac{1}{4}(1-1 / e) \delta_{0}$, which is in turn greater than $\frac{1}{7} \delta_{0}$.

We can now combine Lemmas 7 and 8 into the following:

Lemma 9 Let $X_{1}, X_{2}, \ldots$ be finite non-negative independent identically distributed random variables whose common distribution is not concentrated on a point. Define $S_{n}=\sum_{i=1}^{n} X_{i}$. Fix $c>0$. Then there is a constant $\delta$, such that for any $\epsilon>0$, there exists $n=O(\log (1 / \epsilon))$, such that $\operatorname{Pr}\left[S_{n}<t-c \mid S_{n}<t\right]>\delta$ whenever $\operatorname{Pr}\left[S_{n}<t\right]>\epsilon$.

Proof: Use Lemma 7 to group the $X_{i}$ together into partial sums $Y_{i}=\sum_{j=i n_{0}+1}^{i n_{0}+n_{0}} X_{j}$ with the property that for some $t \operatorname{Pr}\left[Y_{i}<t\right]<\frac{1}{2}$ but $\operatorname{Pr}\left[Y_{i}<t-c\right]=\delta_{0}>0$. (Note that $n_{0}$ does not depend on $\epsilon$, so it disappears into the constant factor.) Then apply Lemma 8 to sums of these $Y_{i}$ variables to get the full result.

\subsection{When the Race Ends}

In this section, we show that a race between $n$ independent delayed renewal processes with bounded added delays ends in $O(\log n)$ rounds with at least constant probability. In the following section, we translate this result, which appears as Corollary 11, back into terms of the LEAN-CONSENSUS algorithm to get Theorem 12 .

Theorem 10 Let $\left\{X_{i j}\right\}$, where $i, j \geq 1$, be a two-dimensional array of finite non-negative independent identically distributed random variables with a common distribution function $F$ that is not concentrated on a point. Let $\left\{\Delta_{i j}\right\}$, where $i \geq 1, j \geq 0$, be a two-dimensional array of constants with $0 \leq \Delta_{i j} \leq M$ when $j \geq 1$. Let $\left\{H_{i j}\right\}$, where $i, j \geq 1$, be a two-dimensional array of independent random variables, each of which is equal to $\infty$ with probability $h(n)$ and 0 otherwise. Define

$$
S_{i r}^{\prime}=\Delta_{i 0}+\sum_{j=1}^{r}\left(\Delta_{i j}+X_{i j}+H_{i j}\right) .
$$

Assume that for any finite $t$, integer $r$, and $i \neq j, \operatorname{Pr}\left[S_{i r}^{\prime}=S_{j r}^{\prime}=t\right]=0$. Let $c$ be any integer constant greater than 0 .

Then there exists a constant $\delta>0$, such that for any $n$, there exists $r=O(\log n)$ and $t$, such that

$$
\operatorname{Pr}\left[\forall i S_{i r}^{\prime}=\infty \vee\left(\exists i \leq n: S_{i, r+c}^{\prime}<t \wedge \forall i^{\prime} \neq i, i^{\prime} \leq n: S_{i^{\prime} r}^{\prime}>t\right)\right]>\delta .
$$


The constant factor in $r=O(\log n)$ and the constant $\delta$ may depend on $c, F, M$, and $h$; but neither constant depends on $n$.

Proof: Since each $X_{i j}$ is finite with probability 1, there exists some constant $c_{1}$ such that $\operatorname{Pr}\left[\sum_{j=r+1}^{r+c} X_{i j}<c_{1}\right]>\frac{1}{2}$. Let $T_{i r}=\sum_{j=1}^{r} X_{i j}$ and let $S_{i r}=T_{i r}+\sum_{j=0}^{r} \Delta_{i r}$. Apply Lemma 9 to the sequence $X_{i j}$ with $c=$ $c M+c_{1}$ and $\epsilon=n^{-2}$ to obtain $r=O(\log n)$ and a constant $\delta_{0}$ for which $\operatorname{Pr}\left[T_{i r}<t-c M-c_{1} \mid T_{i r}<t\right]>\delta_{0}$ whenever $\operatorname{Pr}\left[T_{i r}<t\right]>n^{-2}$. Adding the missing constant terms $\sum_{j=0}^{r} \Delta_{i j}$ to $T_{i r}$ to get $S_{i r}$ is equivalent to subtracting these same terms from each occurrence of $t$, so we in fact have $\operatorname{Pr}\left[S_{i r}<\right.$ $\left.t-c M-c_{1} \mid S_{i r}<t\right]>\delta_{0}$ whenever $\operatorname{Pr}\left[S_{\text {ir }}<t\right]>n^{-2}$. This gives us our target round $r$.

Now apply Lemma 6 to $S_{i r}^{\prime}$, for all $i \leq n$, to show that with probability at least $1 / 5$ either $\forall i S_{i r}^{\prime}=\infty$ or there exists a time $t_{0}$, such that there is a unique winner $i \leq n$ for which $S_{i r}^{\prime}$ is less than $t_{0}$. Let us assume without loss of generality that $n$ is at least 6 . Throw out all cases where $i$ has $\operatorname{Pr}\left[S_{i r}^{\prime}<\right.$ $\left.t_{0}\right] \leq n^{-2}$; this leaves a probability of at least $1 / 5-1 / n \geq 1 / 30$ that (a) there is a unique winner $i$, and (b) $i$ satisfies the condition $\operatorname{Pr}\left[S_{i r}^{\prime}<t_{0}\right]>n^{-2}$, implying $\operatorname{Pr}\left[S_{i r}=S_{i r}^{\prime}<t_{0}\right]>n^{-2}$ and thus $\operatorname{Pr}\left[S_{i r}<t_{0}-c M-c_{1} \mid S_{i r}<\right.$ $\left.t_{0}\right]>\delta_{0}$. So with probability at least $\frac{1}{30} \delta_{0}$, we have $S_{i r}<t_{0}-c M-c_{1}$, and thus with probability at least $\frac{1}{60} \delta_{0}$ we have $S_{i, r+c}<S_{i r}+c M+c_{1}=$ $S_{i r}^{\prime}+c M+c_{1}<t_{0}$.

Suppose that this event holds. It is still possible for $S_{i, r+c}^{\prime}$ to be infinite if $\sum_{j=r+1}^{r+c} H_{i j}=\infty$. Call this event $I$; if $\operatorname{Pr}[I]=1-(1-h(n))^{c}>\frac{1}{120} \delta_{0}$, then $h(n)$ is bounded below by a constant and there exists $r^{\prime}=O(\log n)$ such that $\operatorname{Pr}\left[\forall i S_{i r^{\prime}}^{\prime}=\infty\right]$ is at least a constant. Alternatively, we have $\operatorname{Pr}\left[S_{i, r+c}^{\prime}=S_{i, r+c}<S_{i r}^{\prime}+c M+c_{1}\right]>\delta=\frac{1}{120} \delta_{0}$. In either case, the theorem holds.

Corollary 11 Let $R$ be the first round for which either

- There exists $i$, such that $S_{i, R+c}^{\prime}<S_{i^{\prime} R}^{\prime}$ for all $i^{\prime} \neq i$, or

- For all $i, S_{i, R+c}^{\prime}=\infty$.

Under the conditions of the preceding theorem, $\mathrm{E}[R]=O(\log n)$, and, for any $k \geq 0, \operatorname{Pr}[R>k] \leq e^{-\lfloor k / O(\log n)\rfloor}$.

Proof: Theorem 10 says that the desired event occurs with constant probability $\delta$ after a phase consisting of $r=O(\log n)$ rounds. If it does not occur, we can apply the theorem again to the subset of the $i$ 's for which 
$S_{i, r+c}^{\prime}$ is finite, starting with round $r+c+1$ and setting the initial delay $\Delta_{i 0}$ to the value of $S_{i, r+c}^{\prime}$ from the previous phase.

On average, at most $1 / \delta=O(1)$ such phases are needed, giving $\mathrm{E}[R] \leq$ $(1 / \delta) r=O(\log n)$. For the exponential tail bound, observe that the probability that the algorithm runs for more than $c$ phases of $r$ rounds each is at $\operatorname{most}(1-\delta)^{c}=\left((1-\delta)^{1 / \delta}\right)^{c \delta} \leq\left(e^{-1}\right)^{c \delta}=e^{-c \delta}$. So the probability that the algorithm runs for more than $k$ rounds is at most $e^{-\lfloor k / r\rfloor \delta} \leq e^{-\lfloor k / O(\log n)\rfloor}$.

\subsection{When LEAN-CONSENSUS Ends}

Translating Corollary 11 back into terms of the LEAN-CONSENSUS algorithm gives:

Theorem 12 Under the noisy scheduling model with random failures, starting from any reachable state in the LEAN-CONSENSUS algorithm in which the largest round number of any process is $r$, the algorithm running with $n$ active processes terminates by round $r+r^{\prime}$, where $r^{\prime}$ has expected value $O(\log n)$ and $\operatorname{Pr}\left[r^{\prime}>k\right] \leq e^{-\lfloor k / O(\log n)\rfloor}$ for any $k \geq 0$.

Proof: Apply Corollary 11 with $c=2$ and the initial delays $\Delta_{i 0}$ set to the times at which each process completes round $r$. This shows that after $R$ additional rounds, where $\mathrm{E}[R]=O(\log n)$ and $\operatorname{Pr}[R>k] \leq e^{-\lfloor k / O(\log n)\rfloor}$, either some process $P$ finishes some round $s$ before any other process finishes round $s-2$, or all processes fail. In the first case, if $P$ prefers $b$, it is the only process to have written to $a_{b}[s-1]$ or $a_{1-b}[s-1]$ by the time it reads $a_{1-b}[s-1]$ as part of round $s$. Thus it reads a zero from $a_{1-b}[s-1]$ and decides. All other processes decide at most one round later by Lemma 4 . We thus get $r^{\prime} \leq R+1$, and the single extra round disappears into the constant factors.

It is not hard to see that an $O(\log n)$ bound is the best possible, up to constant factors.

Theorem 13 There exists a noise distribution $F$ and a set of delays $\Delta$ such that the LEAN-CONSENSUS algorithm requires expected $\Omega(\log n)$ rounds in the noisy scheduling model, even without failures.

Proof: Let all $\Delta_{i j}=0$ for $j>0$, and let $F$ have each operation take either 1 or 2 time units with equal probability. Then any single processor completes its first $\log n$ operations in 1 time unit each with probability $1 / n$. 
To avoid simultaneous operations, let $\Delta_{i 0}$ be some small distinct epsilon value for each $i$.

Start $n / 2$ processes with input 0 and $n / 2$ with input 1 . The probability that there exists at least one 0 -input process and at least one 1 -input process that both complete their first $\log n$ operations in 1 time unit each is given by

$$
\left(1-\left(1-\frac{1}{n}\right)^{n / 2}\right)^{2}
$$

which goes to $\left(1-e^{-1 / 2}\right)^{2}=\Theta(1)$ in the limit as $n$ grows. So there is a constant probability that at least one process with each input runs for $\log n$ operations without ever changing its preference to that of a faster process with the opposite preference, and we get expected $\Omega(\log n)$ rounds of disagreement.

\section{Termination with Quantum and Priority-Based Scheduling}

In this section, we consider the question of termination subject to hybrid quantum and priority-based scheduling on a uniprocessor. The required quantum size is 8 operations; curiously, this is the same size required for the specialized algorithm given in [5]. We see this coincidence as hinting at the possibility that all shared-memory consensus algorithms may ultimately converge to a single ideal algorithm (though such an ideal algorithm, if it exists, is probably not identical to LEAN-CONSENSUS).

Theorem 14 When running LEAN-CONSENSUs in a hybrid-scheduled system with a quantum of at least 8 operations, every process decides after executing at most 12 operations.

Proof: We will show that at most one of $a_{0}[1]$ and $a_{1}[1]$ is set before some process finishes round 2 and decides. Consider an execution in which $a_{0}[1]$ and $a_{1}[1]$ are each set at some point. Let $P_{0}$ and $P_{1}$ be the first processes to set $a_{0}[1]$ and $a_{1}[1]$, respectively. Neither $P_{0}$ nor $P_{1}$ can have observed the round-1 write of the other, or it would have changed its preference. Thus both processes' round-1 reads of $a_{0}[1]$ and $a_{1}[1]$ must have occurred before either performed its round-1 write. Since we are on a uniprocessor, this can only occur if one of the processes was pre-empted before its write occurred. 
Assume without loss of generality that $P_{0}$ is this unlucky process. Since $P_{0}$ is the first process to write to $a_{0}[1]$, if we can show that $P_{0}$ is not rescheduled before some process completes round 2 , then that process decides 1 (and by Lemma 4, all processes eventually decide 1) as soon as it observes a zero in $a_{0}[1]$. So we need only show that $P_{0}$ is not rescheduled until some other process completes eight operations.

Let $Q_{1}$ be the process that pre-empts $P_{0}$. At the time of pre-emption, $Q_{1}$ is at the start of a quantum; it either finishes eight operations without being pre-empted or is pre-empted by a higher-priority process $Q_{2}$. But $Q_{2}$ in turn can only be pre-empted before completing its quantum by some higher-priority process $Q_{3}$. After at most $n$ such pre-emptions, we run out of higher-priority processes, and the last process runs to the end of its quantum and decides. Note that all of the processes in this chain (except possibly $Q_{1}$ ) have a higher priority than $P_{0}$ and thus cannot be equal to $P_{0}$. It follows that some process finishes round 2 before $P_{0}$ is rescheduled, and thus every process decides 1 by the end of round 3 .

\section{Bounded space LEAN-CONSENSUS}

The LEAN-CONSENSUS algorithm as described in Section 4 requires infinite space. In this section, we describe how to modify the algorithm to use bounded space. We assume that we have available a backup protocol, which is a bounded-space consensus protocol that requires polynomial work per process (for example, the $O\left(n^{4}\right)$ protocol in [6] works). We will build a protocol that combines LEAN-CONSENSUS with the backup protocol in a way that only uses the backup protocol rarely, so that its high cost adds only a constant to the $O(\log n)$ cost of the combined protocol.

Note that such a combined protocol is not necessary in the model of Section 7, as in that model we only need space for 3 rounds of LEAN-CONSENSUS.

The combined protocol operates as follows:

1. Run LEAN-CONSENSUS through round $r_{\max }$.

2. At round $r_{\max }+1$, switch to the backup protocol, using the preference at the end of round $r_{\max }$ of LEAN-CONSENSUS as input to the backup protocol.

If $r_{\max }$ is large enough, most of the time we will expect that LEANCONSENSUS terminates before reaching $r_{\max }$ and the backup algorithm will 
not be used. But in the case where $r_{\max }$ is reached (say, because the scheduler is nastier than we have assumed), the backup algorithm guarantees termination using bounded space and bounded (but possibly very large) expected time.

Theorem 15 For any polynomial-work consensus protocol chosen as a backup algorithm and any noise distribution, there is a choice of $r_{\max }=O\left(\log ^{2} n\right)$ such that the combined algorithm described above is a consensus protocol that requires $O(\log n)$ expected operations per process and $O\left(\log ^{2} n\right)$ bits in the $a_{0}$ and $a_{1}$ arrays.

Proof: First let us show that the combined algorithm solves consensus. Validity is immediate from Lemma 3; when all inputs are equal, we never get past round 2 and the combined algorithm behaves identically to LEANCONSENSUS. For agreement, the only tricky case is when some processes decide during LEAN-CONSENSUS and others decide during the backup protocol. But if some process $P$ decides $b$ at or before round $r$, then by Lemmas 2 and 4 no process writes $a_{1-b}[r]$ and every process that executes the backup protocol has $b$ as input. Thus the validity condition for the backup protocol implies that all processes decide $b$.

Now let us show that there is a choice of $r_{\max }$ that gives the desired performance bound. Suppose each process finishes the backup protocol in $O\left(n^{c}\right)$ expected operations. By Theorem 12, there is a value $T=O(\log n)$ such that the probability that LEAN-CONSENSUS does not finish by round $k$ is at most $e^{-\lfloor k / T\rfloor}$. Setting $r_{\max }=T \cdot c \cdot \log n=O\left(\log ^{2} n\right)$, the backup protocol is run with probability at most $e^{-c \log n}=n^{-c}$, and thus it contributes at most $n^{-c} O\left(n^{c}\right)=O(1)$ to the expected cost.

Finally, the size of the $a_{0}$ and $a_{1}$ arrays is clearly equal to $r_{\max }=$ $O\left(\log ^{2} n\right)$.

\section{Simulation Results}

Figure 11 gives the results of simulating LEAN-CONSENSUS with various interarrival distributions. These simulations are of the model as described in Section 3.1; in particular it is assumed that all operations take zero time and that there are no contention effects or synchronization issues.

The $\mathrm{X}$ axis is plotted on a logarithmic scale and represents the number of processes. The $\mathrm{Y}$ axis is plotted on a linear scale and represents the round at which the first process terminates (which may be one less than the round 
Mean round of first termination

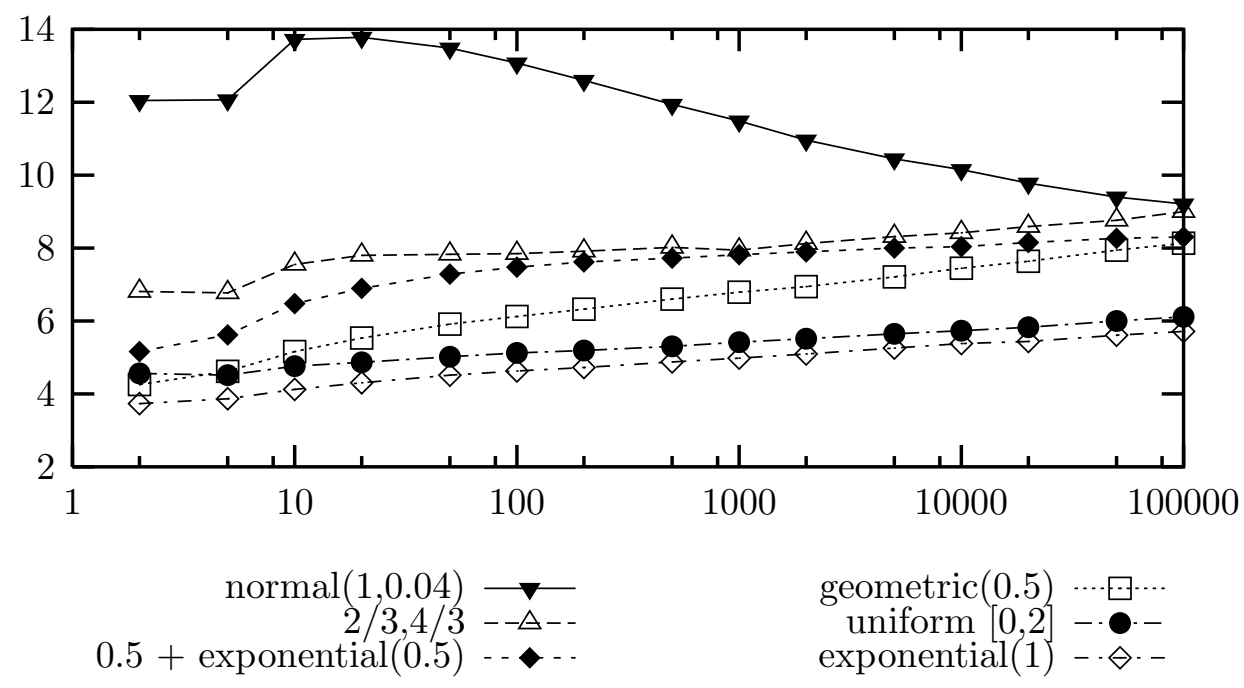

Figure 1: Results of simulating LEAN-CONSENSUS with various interarrival distributions.

at which the last process terminates). Each point in the graph represents an average termination round in 10,000 trials with the given distribution and number of processes. The starting times for all processes are the same except for a small random epsilon, generated uniformly in the range $\left(0,10^{-8}\right)$. In each case, half the processes are started with input 0 and half with input 1 . There are no failures.

The random number generator used was drand48. The distributions used were:

1. Normal distribution with mean 1 and standard deviation 0.2 (variance $0.04)$, rejecting points outside $(0,2)$.

2. $2 / 3$ or $4 / 3$ with equal probability.

3. 0.5 plus an exponential random variable with mean 0.5. This corresponds to a delayed Poisson process.

4. Geometric with $p=0.5$.

5. Uniform in $(0,2)$. 
6. Exponential with mean 1. This corresponds to a Poisson process with no initial delay; it is also equivalent to generating a schedule by choosing one process uniformly at random for each time unit.

It is worth noting that while the expected number of rounds grows logarithmically for most distributions, both the rate of growth and the initial value are small. These small constant factors may be the result of most processes adopting the values of early leaders, so that termination can be reached by agreement among leaders rather than the emergence of a single leader.

The inverted behavior with a normal distribution is intriguing; it suggests that with large numbers of processes there are more chances for one particularly speedy process to leap ahead of its competitors, and that for some distributions this effect overshadows the effect of having more competitors to leap ahead of. It is not clear from the data whether this curve eventually turns around and starts rising again, or whether it converges to some constant asymptote.

\section{Conclusions, Extensions, and Future Work}

We see this paper as making two main contributions. The first is the extraction of the adaptive $\Theta(\log n)$ time LEAN-CONSENSUS protocol from its more sophisticated predecessors and the demonstration that this simplified algorithm can solve consensus in models that are less extreme than those predecessors were designed to survive but that are perhaps closer to capturing the scheduling behavior an algorithm is likely to experience in practice. Although LEAN-CONSENSUS does not really contain any new ideas, we believe that ripping out features that practitioners might balk at implementing is a valuable task in its own right.

The second is the noisy scheduling model. This model limits the adversary not by covering its eyes but by making its hands shake. It allows us to express the understanding that in the real world failures and timing are usually not fully under the control of intelligent demons, while still retaining a healthy respect for the subtlety and unpredictability of the world. We believe that this "perturbed worst-case analysis" approach is likely to have applications in many areas both in and outside of distributed computing.

There are still many questions left unanswered and many ways in which the noisy scheduling model could be extended. We discuss some of these issues below. 
Non-random failures. It would be nice to understand how LEAN-CONSENSUS fares with failures that are not random. We can get an upper bound in this situation by restarting Theorem 12 whenever a process dies. Since the adversary must kill at least one process every expected $O(\log n)$ rounds, the algorithm terminates in expected $O(f \log n)$ rounds where $f$ is the number of failures. This bound compares favorably with the $O\left(n \log ^{2} n\right)$ work per processor needed by the best known randomized algorithm that solves consensus with a fully-adaptive adversary and up to $n-1$ failures [9], but the fullyadaptive adversary is much stronger than one limited to noisy scheduling. It seems likely that a better upper bound than $O(f \log n)$ could be obtained by a more careful analysis that includes how processes change preferences. We conjecture that the real bound is in fact $O(\log n)$.

Statistical adversaries. We would also like to do away with the fixed bound $M$ on the delay between operations under the control of the adversary. The technical reason for including this bound in the model is that it provides a scale for the noise introduced by the $X_{i j}$ variables; if the adversary can increase $\Delta_{i j}$ without limit, it can construct a steadily slower and slower execution in which the noise, relative to the gap between rounds, never accumulates enough to affect the schedule. But a weaker statistical constraint, such as requiring $\sum_{j=1}^{r} \Delta_{i j} \leq r M$, might avoid such Zeno-like pathologies while allowing more variation in the gaps between operations. 9 The present proof does not work with just this statistical constraint (the particular step that breaks down is the use of Lemma 9 to show that being ahead at round $r$ often means being ahead by $c$ at round $r$ ), but we conjecture that the statistical constraint is in fact enough to get termination in $O(\log n)$ rounds.

Synchronization and contention. Though the present work was motivated by a desire to move away from powerful theoretical adversaries toward a model more closely reflecting the non-maliciousness of misbehavior in real systems, we cannot claim that the model accurately describes the behavior of any real shared-memory system. One difficulty is that real shared-memory systems generally do not guarantee full serializability of memory operations in the absence of additional synchronization operations (see [28, Section 8.6]). We can overcome this difficulty by adding synchronization barriers to each round of LEAN-CONSENSUS; in principle this does not affect the analysis since the structure of each round is still the same as all other rounds. A

\footnotetext{
${ }^{5}$ This is a bit like using the statistical adversary of [18].
} 
second problem is memory contention, which we have not analyzed. The difficulty with both explicit synchronization and memory contention is that their effects are unlikely to be consistent with the assumption that the timing of different processes' operations are independent. To the extent that this lack of independence disperses processes (say, by slowing down laggards fighting over congested early-round registers while allowing the speedy to sail through relatively clear late-round registers), it helps the algorithm. Whether such an effect would occur in practice cannot easily be predicted without experimentation.

Lower bounds. The noisy scheduling model is friendly enough that an $O(\log n)$ running time for consensus might not be the best possible. A counterexample like the one given in the proof of Theorem 13 might be able to show that no deterministic algorithm with certain strong symmetry properties (such as no dependence on process identity and a mirror-image handling of the different inputs) can do better, but it not obvious where to look for a more general lower bound. It is not out of the question that a clever algorithm could solve consensus with noisy scheduling in as little as $O(1)$ time.

Message passing. All of our results are set in a shared-memory model. It would be interesting to see whether a noisy scheduling assumption can be used to solve consensus quickly in an asynchronous message-passing model.

Other problems. Finally, though we have concentrated on a particularly simplified protocol for solving a single fundamental problem, it would be interesting to see how other algorithms fare in the noisy scheduling model. It seems likely, for example, that algorithms designed for unknown-delay models such as Alur et al.'s [3] should continue to work in the noisy scheduling model, perhaps with some constraint on the noise distribution to exclude random delays with unbounded expectations. Similarly the line of inquiry started by Gafni and Mitzenmacher [23], on analyzing the behavior of timing-based algorithms for mutual exclusion and related problems with random scheduling, could naturally extend to the more general model of noisy scheduling. 


\section{Acknowledgments}

I would like to thank Faith Fich and Maurice Herlihy for insightful comments on the plausibility of an early version of the noisy scheduling model; the remaining implausibility is my fault and not theirs. I am also indebted to Robbert van Renesse for pointing out the "narrowness" of the bad execution paths needed to prevent consensus as a reason for the relative lack of concern for asynchronous impossibility results among practitioners.

\section{References}

[1] Karl R. Abrahamson. On achieving consensus using a shared memory. In Proceedings of the Seventh Annual ACM Symposium on Principles of Distributed Computing, pages 291-302, Toronto, Ontario, Canada, 15-17 August 1988.

[2] Yehuda Afek, Dalia Dauber, and Dan Touitou. Wait-free made fast (extended abstract). In Proceedings of the Twenty-Seventh Annual ACM Symposium on the Theory of Computing, pages 538-547, Las Vegas, Nevada, 29 May-1 June 1995.

[3] Rajeev Alur, Hagit Attiya, and Gadi Taubenfeld. Time-adaptive algorithms for syncronization. SIAM Journal on Computing, 26(2):539-556, April 1997.

[4] J. H. Anderson, R. Jain, and D. Ott. Wait-free synchronization in quantum-based multiprogrammed systems. In Distributed Computing; 12th International Symposium; Proceedings, volume 1499 of Lecture Notes in Computer Science, pages 34-45, Andros, Greece, September 1998. Springer-Verlag.

[5] James H. Anderson and Mark Moir. Wait-free syncronization in multiprogrammed systems: Integrating priority-based and quantum-based scheduling. In Proceedings of the Eighteenth Annual ACM Symposium on Principles of Distributed Computing, pages 123-132, Atlanta, Georgia, USA, 3-6 May 1999.

[6] James Aspnes. Time- and space-efficient randomized consensus. Journal of Algorithms, 14(3):414-431, May 1993.

[7] James Aspnes. Lower bounds for distributed coin-flipping and randomized consensus. Journal of the ACM, 45(3):415-450, May 1998. 
[8] James Aspnes and Maurice Herlihy. Fast randomized consensus using shared memory. Journal of Algorithms, 11(3):441-461, September 1990.

[9] James Aspnes and Orli Waarts. Randomized consensus in expected $O\left(N \log ^{2} N\right)$ operations per processor. SIAM Journal on Computing, 25(5):1024-1044, October 1996.

[10] Hagit Attiya, Danny Dolev, and Nir Shavit. Bounded polynomial randomized consensus. In Proceedings of the Eighth Annual ACM Symposium on Principles of Distributed Computing, pages 281-293, Edmonton, Alberta, Canada, 14-16 August 1989.

[11] Hagit Attiya and Arie Fouren. Adaptive wait-free algorithms for lattice agreement and renaming (extended abstract). In Proceedings of the Seventeenth Annual ACM Symposium on Principles of Distributed Computing, pages 277-286, Puerto Vallarta, Mexico, 28 June-2 July 1998.

[12] Yonatan Aumann. Efficient asynchronous consensus with the weak adversary scheduler. In Proceedings of the Sixteenth Annual ACM Symposium on Principles of Distributed Computing, pages 209-218, Santa Barbara, California, 21-24 August 1997.

[13] Yonatan Aumann and Michael A. Bender. Efficient asynchronous consensus with the value-oblivious adversary scheduler. In Friedhelm Meyer auf der Heide and Burkhard Monien, editors, Automata, Languages and Programming, 23rd International Colloquium, volume 1099 of Lecture Notes in Computer Science, pages 622-633, Paderborn, Germany, 8-12 July 1996. Springer-Verlag.

[14] Patrick Billingsley. Probability and Measure. John Wiley and Sons, second edition, 1986.

[15] Gabriel Bracha and Ophir Rachman. Randomized consensus in expected $O\left(n^{2} \log n\right)$ operations. In Sam Toueg, Paul G. Spirakis, and Lefteris M. Kirousis, editors, Distributed Algorithms, 5th International Workshop, volume 579 of Lecture Notes in Computer Science, pages 143-150, Delphi, Greece, 7-9 October 1991. Springer, 1992.

[16] Tushar Deepak Chandra. Polylog randomized wait-free consensus. In Proceedings of the Fifteenth Annual ACM Symposium on Principles of Distributed Computing, pages 166-175, Philadelphia, Pennsylvania, USA, 23-26 May 1996. 
[17] Benny Chor, Amos Israeli, and Ming Li. Wait-free consensus using asynchronous hardware. SIAM Journal on Computing, 23(4):701-712, August 1994.

[18] Andrew Chou, Jeremy Cooperstock, Ran El-Yaniv, Michael Klugerman, and Tom Leighton. The statistical adversary allows optimal money-making trading strategies. In Proceedings of the Sixth Annual ACM-SIAM Symposium on Discrete Algorithms, pages 467-476, San Francisco, California, 22-24 January 1995.

[19] Richard Cole and Ofer Zajicek. The expected advantage of asynchrony. Journal of Computer and System Sciences, 51(2):286-300, October 1995.

[20] Danny Dolev, Cynthia Dwork, and Larry Stockmeyer. On the minimal synchronism needed for distributed consensus. Journal of the ACM, 34(1):77-97, January 1987.

[21] Cynthia Dwork, Nancy Lynch, and Larry Stockmeyer. Consensus in the presence of partial synchrony. Journal of the ACM, 35(2):288-323, April 1988.

[22] Michael J. Fischer, Nancy A. Lynch, and Michael S. Paterson. Impossibility of distributed consensus with one faulty process. Journal of the ACM, 32(2):374-382, April 1985.

[23] Eli Gafni and Michael Mitzenmacher. Analysis of timing-based mutual exclusion with random times. In Proceedings of the Eighteenth Annual ACM Symposium on Principles of Distributed Computing, pages 13-21, Atlanta, Georgia, USA, 3-6 May 1999.

[24] Oded Goldreich and Erez Petrank. The best of both worlds: Guaranteeing termination in fast randomized byzantine agreement protocols. Information Processing Letters, 36(1):45-49, 1990.

[25] Elias Koutsoupias and Christos H. Papadimitriou. Beyond competitive analysis. In 35th Annual Symposium on Foundations of Computer Science, pages 394-400, Santa Fe, New Mexico, 20-22 November 1994. IEEE.

[26] Leslie Lamport. A fast mutual exclusion algorithm. ACM Transactions on Computer Systems, 5(1):1-11, February 1987. 
[27] Michael C. Loui and Hosame H. Abu-Amara. Memory requirements for agreement among unreliable asynchronous processes. In Franco P. Preparata, editor, Advances in Computing Research, volume 4. JAI Press, 1987.

[28] David A. Patterson, John L. Hennessy, and David Goldberg. Computer Architecture: A Quantitative Approach. Morgan Kaufmann Publishers, 2nd edition, 1996.

[29] Srikanth Ramamurthy, Mark Moir, and James H. Anderson. Real-time object sharing with minimal system support (extended abstract). In Proceedings of the Fifteenth Annual ACM Symposium on Principles of Distributed Computing, pages 233-242, Philadelphia, Pennsylvania, USA, 23-26 May 1996.

[30] Michael Saks, Nir Shavit, and Heather Woll. Optimal time randomized consensus - making resilient algorithms fast in practice. In Proceedings of the Second Annual ACM-SIAM Symposium on Discrete Algorithms, pages 351-362, San Francisco, California, 28-30 January 1991. 